\title{
Exploring targets and signaling pathways of paeonol involved in relieving inflammation based on modern technology
}

\author{
Jian-hong Qi ${ }^{1}\left(\mathbb{D} \cdot\right.$ Fang-xu Dong ${ }^{2} \cdot$ Xiao-long Wang $^{3,4,5}$
}

Received: 29 June 2021 / Accepted: 19 August 2021 / Published online: 31 August 2021

(c) The Author(s), under exclusive licence to Springer Nature Switzerland AG 2021

\begin{abstract}
Paeonol, derived from natural plants (Moutan Cortex), has a wide range of biological effects, including anti-inflammatory and antitumor effects as well as favorable effects against cardiovascular and neurodegenerative diseases. The anti-inflammatory action is the main pharmacological activity of paeonol and has the greatest clinical relevance. However, the anti-inflammatory mechanism of paeonol has not been reported in sufficient detail. We systematically analyzed the anti-inflammatory mechanism of paeonol using network pharmacological databases and platforms, including TCMSP, Swiss TargetPrediction, OMIM, DrugBank, TTD, Jevnn, STRING11.0, and Metascape. Furthermore, we used high-throughput molecular docking method to prove the results of the above analyses, providing a reference for exploring the mechanism of paeonol and developing targeted drugs.
\end{abstract}

\section{Graphic abstract}
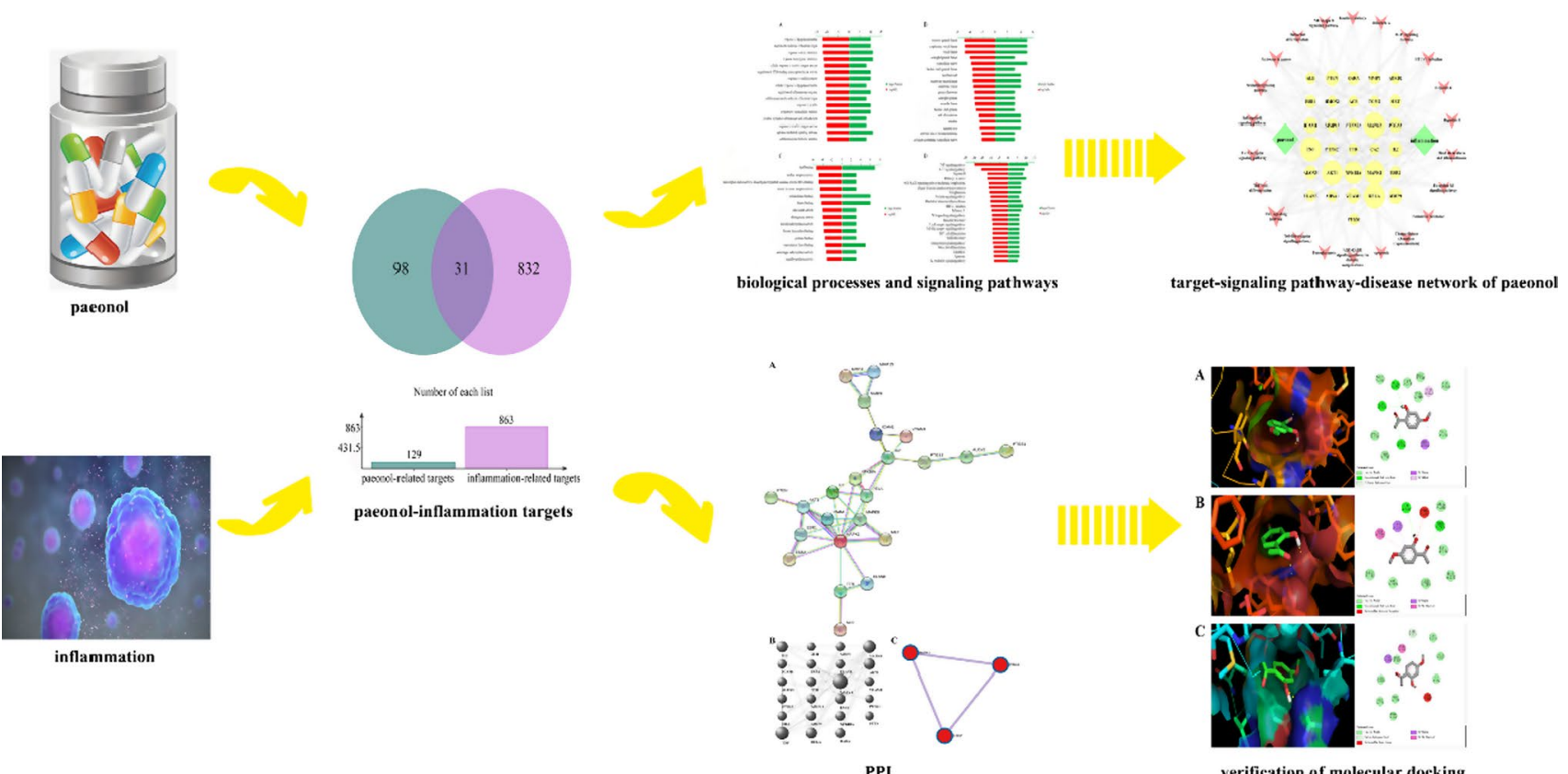

verification of molecular docking

Keywords Paeonol $\cdot$ Inflammation $\cdot$ Network pharmacology $\cdot$ Molecular docking $\cdot$ Target

Jian-hong Qi and Fang-xu Dong have contributed equally to this work.

Extended author information available on the last page of the article

\section{Abbreviations}

SUV Solar ultraviolet

TOPK T-LAK cell-derived protein kinase

MAPKs Mitogen-activated protein kinase 


$\begin{array}{ll}\text { ERK } & \text { Extracellular regulated protein kinase } \\ \text { ERBB2 } & \text { Epidermal growth factor receptor 2 } \\ \text { PPI } & \text { Protein-protein interaction network } \\ \text { GO } & \text { Gene ontology } \\ \text { KEGG } & \text { Kyoto encyclopedia of genes and genomes } \\ \text { BP } & \text { Biological Processes } \\ \text { CC } & \text { Cellular components } \\ \text { MF } & \text { Molecular functions } \\ \text { MMP9 } & \text { Matrix metalloproteinase-9 } \\ \text { TNF } & \text { Tumor necrosis factor }\end{array}$

\section{Introduction}

Paeonol has shown many pharmacological effects in various experiments in vivo and in vitro. In addition to antiinflammatory and antitumor effects, it positively influences cardiovascular and neurodegenerative diseases. Paeonol has been shown to alleviate solar ultraviolet (SUV)-induced skin inflammation by acting on T-LAK cell-derived protein kinase (TOPK) [1], and mitogen-activated protein kinase (MAPKs)/extracellular regulated protein kinase (ERK)/p38 signaling pathway is an important pathway used by paeonol to alleviate specific dermatitis [2]. In cell and molecular experiments, paeonol has significantly inhibited the growth and proliferation of gastric cancer cells and promoted their apoptosis, and the mechanism may be closely related to epidermal growth factor receptor 2 (ERBB2) [3]. Paeonol can prevent atherosclerosis by acting on miR-126 to reduce the formation of low-density lipoprotein [4]. In addition, paeonol can improve neurodegenerative diseases such as Alzheimer's disease and depression by reducing reactive oxygen species (ROS) level, thereby playing a neuroprotective role $[5,6]$.

Studies of anti-inflammatory activity of paeonol began in the sixties of the twentieth century [7]. According to previous research findings, the anti-inflammatory effect of paeonol is its most prominent pharmacological effect. To further increase the understanding of the anti-inflammatory activity and the development of targeted drugs, we explored the antiinflammatory mechanism of paeonol by network pharmacology. Moreover, the interactions of paeonol with the core target proteins were virtually verified based on Autodock vina software.

\section{Materials and methods}

\section{Screening of paeonol-related targets}

We searched potential targets of paeonol based on TCMSP (https://tcmspw.com/tcmsp.php) [8] and the Swiss TargetPrediction (http://www.swisstargetprediction.ch/) [9]. We standardized the symbols of target proteins in accordance with the Uniprot protein database (https://www.uniprot.org/) [10].

\section{Screening of inflammation-related targets}

Using the key words related to inflammation, such as "inflammation," "arthritis," "dermatitis," "organ inflammation," "colitis," "periodontitis," and "stomatitis," we screened 863 high-scoring targets for inflammation based on disease databases, including OMIM (https://omim.org/) [11], DrugBank (https://www.drugbank.ca/) [12], and TTD (http://db.idrblab.net/ttd/) [13].

\section{Construction of PPI network for anti-inflammatory targets of paeonol}

To fully understand the interaction between paeonol-related targets and inflammation-related targets, we used the Jevnn platform (http://www.bioinformatics.com.cn/static/others/ jvenn/example.html) [14] to intersect these interactions and create a Venn diagram.

To construct the protein-protein interaction network (PPI) model, we entered the common targets into STRING11.0 (https://string-db.org/) [15].

\section{Enrichment analysis of paeonol-inflammatory targets' function and pathways}

Using Metascape platform (http://metascape.org/gp/index. html) (Zhou et al., 2019) for Gene Ontology (GO) analysis and Kyoto Encyclopedia of Genes and Genomes (KEGG) enrichment (Table 3), we entered the anti-inflammatory targets of paeonol and set the test parameters $(\mathrm{P}<0.01)$ to obtain the main biological processes and signaling pathways.

\section{Construction of paeonol targets signaling pathways network}

Based on Cytoscape software, we drew paeonol targets signaling pathways network to further study the anti-inflammatory mechanism of paeonol.

\section{Virtual verification of molecular docking}

Molecular docking is a virtual drug design technique to verify the interaction between receptor and ligand. We used molecular docking technology to verify the interaction between paeonol and core targets, so as to provide theoretical support for the results of network pharmacology. 


\section{Results}

\section{Screening of paeonol-related targets}

Using "paeonol" as the keyword, we searched and saved 129 possible target proteins of paeonol from the TCMSP and the Swiss TargetPrediction databases.

\section{Screening of inflammation-related targets}

In our previous review of paeonol, we found that the antiinflammatory effect of paeonol mainly involved "arthritis," "dermatitis," "organ inflammation," "colitis," "periodontitis," and "stomatitis." Using these keywords, we found 863 inflammation-related and non-repetitive targets based on the disease databases.

\section{Screening of core targets}

In order to analyze the association network of paeonol anti-inflammatory targets, we had to find the intersection of paeonol-related targets and inflammation-related targets and identified 31 common targets as shown in Fig. 1.

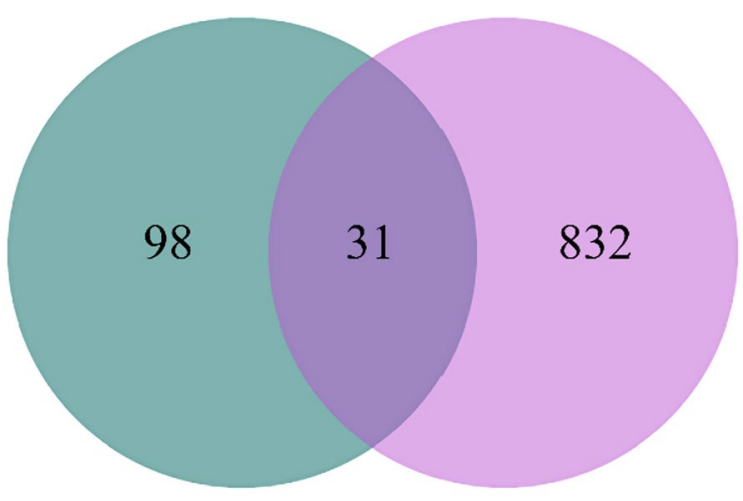

Number of each list

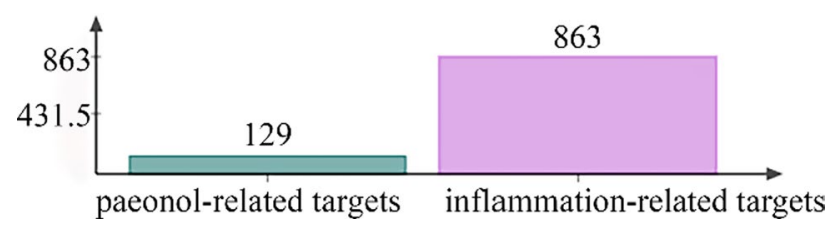

Fig. 1 Venn diagrams of paeonol-related targets and inflammationrelated targets

\section{Construction of PPI network for anti-inflammatory targets of paeonol}

PPI network is an important means to show the importance of targets and their interactions. We constructed PPI network based on STRING11.0 platform and set the minimum interaction threshold (highest confidence $\geq 0.9$ ). Finally, we obtained 23 relatively important targets in Fig. 2: RELA, TNF, AKT1, MAPK8, ALOX5, ESR1, NFKBIA, MMP9, IL2, VCAM1, RARA, ELANE, ALB, MET, ESR2, TTR, MMP3, PTEN, PTGS2, PTGS1, MAPK1, ICAM1, and MMP13. In particular, the module formed by MAPK1, RARA, and ESR2 may have potential biological significance for inflammation.

\section{GO and KEGG analyses}

We annotated and enriched the core targets on Metascape platform, and then analyzed their functions and pathways. GO analysis included the analysis of biological processes (BP), cellular components (CC), and molecular functions (MF). KEGG analysis focused on the enrichment and annotation of signaling pathways involved in paeonol anti-inflammatory actions. As shown in Fig. 3, we obtained 16 BP, 18 $\mathrm{CC}, 14 \mathrm{MF}$, and 22 signaling pathways, and most of these biological processes and signaling pathways are closely related to inflammation. In addition, TNF and IL-17 signaling pathways were the main signaling pathways detected (Table 1). Therefore, these results suggest that paeonol exerts anti-inflammatory effects through multitarget and multisignaling pathways.

\section{Construction of paeonol targets signaling pathways network}

To further explain the anti-inflammatory mechanism of paeonol, we used Cytoscape software to construct paeonol targets signaling pathways network. As shown in Fig. 4 and Table 2, there are 226 edges and 55 nodes in the network. In addition, the network also contains 31 targets and 22 signaling pathways. RELA and MAPK8 are the optimal targets, with the numerical value of degree 22 and the numerical value of closeness centrality 0.613636 ; TNF signaling pathway is the optimal target, with the numerical value of degree 17 and the numerical value of closeness centrality 0.514286 . Therefore, paeonol may exert anti-inflammatory biological effects by acting on 33 main targets and 22 important signaling pathways.

\section{Molecular docking of core targets}

Virtual verification of 23 core targets obtained by PPI was carried out based on Autodock vina software [16]. The 
Fig. 2 PPI network for antiinflammatory targets of paeonol. a Network nodes represent proteins: colored nodes mean the first shell of interactors. Node content: 3D structure of the protein. Edges of different colors represent protein-protein associations: light blue and purple edges mean known interactions (database \& experiment); green, red, and blue edges mean predicted interactions (gene neighborhood, gene fusions, gene co-occurrence); yellow edges mean text mining; and black edges indicate coexpression. b The PPI network was processed by Cytoscape software: larger areas denote more important nodes. c Modules with potential biological significance in PPI

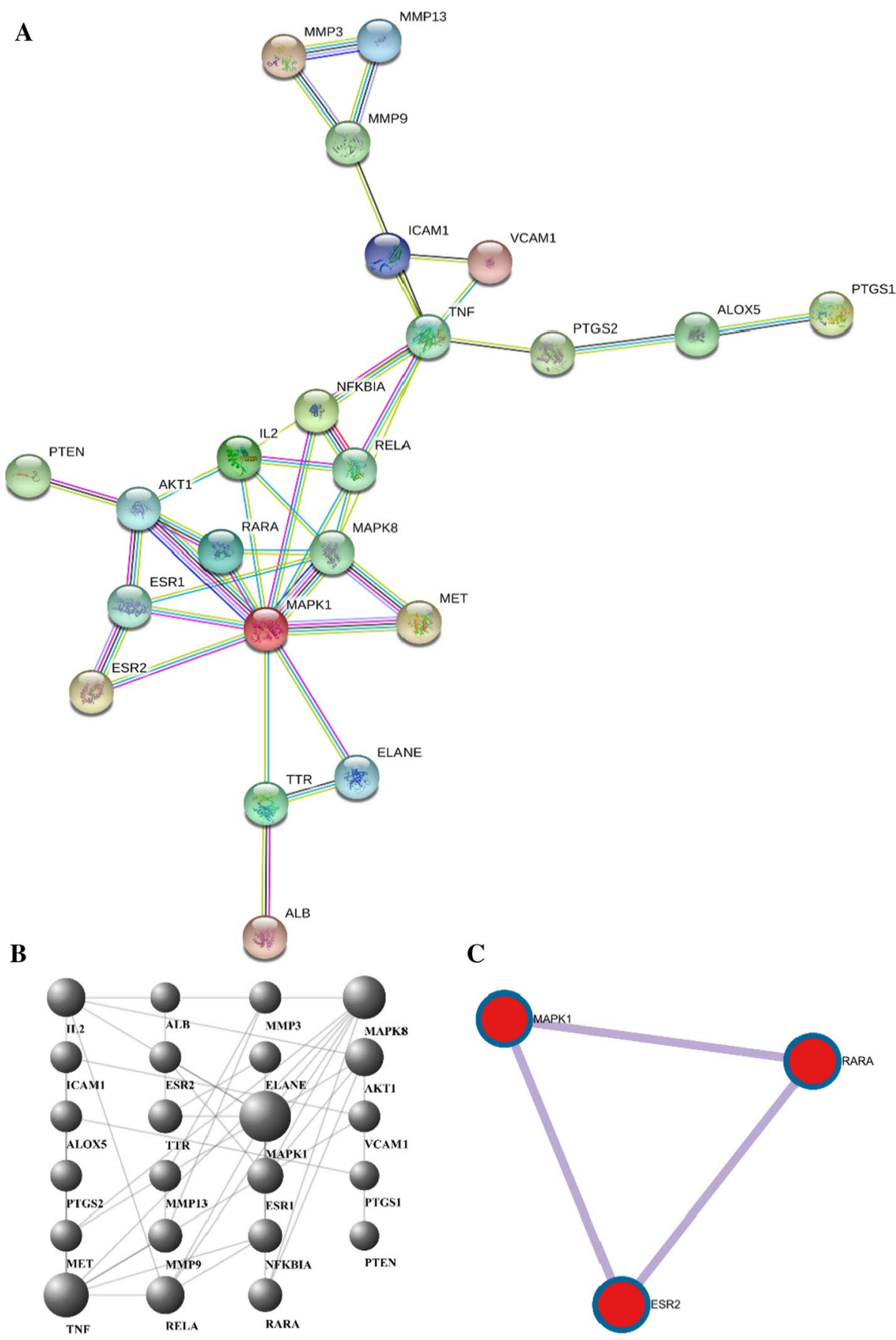

structures of target proteins (.pdb) and paeonol (.mol2) were downloaded from PDB database [17] and TCMSP database, respectively. Importantly, the screening of crystal structures for targets was based on the following principles: containing original ligand, high resolution, and high reliability. Paeonol was subjected to hydrogenation, charging, merging of non-polar hydrogen, and rotating chemical bonds by using AutodockTools software, and it was saved as PDBQT format file. The structures of target proteins were pretreated based on PyMol [18] and AutodockTools software, including hydrogenation, charging, definition of atomic types, merging of non-polar hydrogen, repairing of amino acid residues, removing water, ions, ligands, and excess amino acid chains, and finally saved as PDBQT files. Then, the config files of target proteins were created to set the parameters of grid box. The optimal binding energies between the 23 target proteins and paeonol were calculated by Autodock vina software. Smaller numerical values indicated stronger binding ability. The numerical values with binding energy less than $-5 \mathrm{kcal} /$ mol account for $74 \%$ in Table 3, indicating that paeonol may 


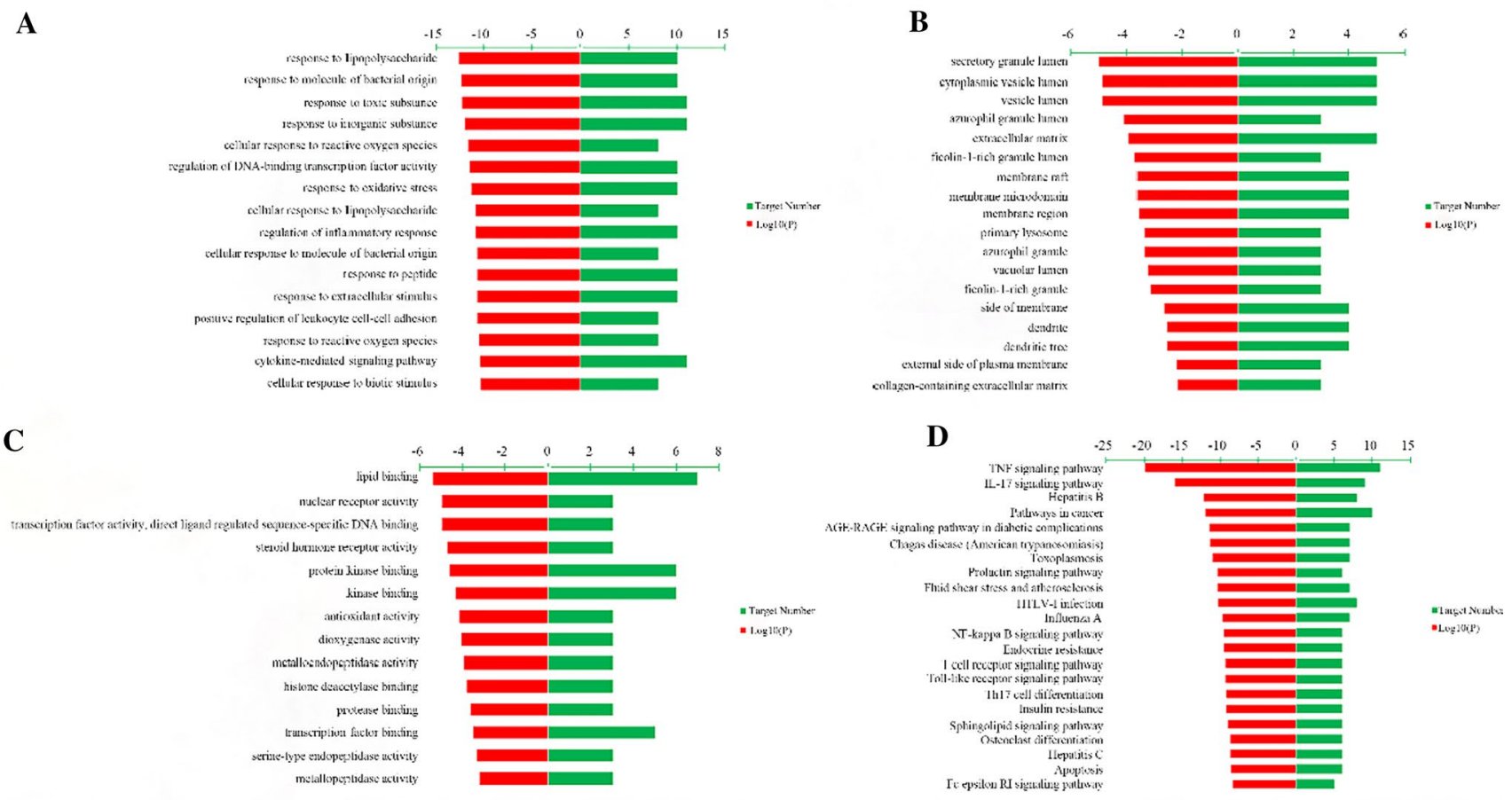

Fig. 3 GO and KEGG analyses of paeonol anti-inflammatory activity. a represents biological processes, $\mathbf{b}$ represents cellular components, $\mathbf{c}$ represents molecular functions, D represents signaling pathways

Table 1 Enrichment information of signaling pathways

\begin{tabular}{|c|c|c|c|}
\hline Signaling pathway & Count & $\log 10(\mathrm{P})$ & Targets \\
\hline TNF signaling pathway & 11 & -20.00294635 & $\begin{array}{l}\text { AKT1|ICAM1|MMP3|MMP9|NFKBIA|MAPK1|MAPK8|PTGS2|REL } \\
\text { AITNF|VCAM1 }\end{array}$ \\
\hline IL-17 signaling pathway & 9 & -16.04429079 & MMP3|MMP9|MMP13|NFKBIA|MAPK1|MAPK8|PTGS2|RELA|TNF \\
\hline Hepatitis B & 8 & -12.25489905 & AKT1|MMP9|NFKBIA|MAPK1|MAPK8|PTEN|RELA|TNF \\
\hline Pathways in cancer & 10 & -11.97434934 & $\begin{array}{l}\text { AKT1|MET|MMP9|NFKBIA|MAPK1|MAPK8|PTENIPTGS2|RARA| } \\
\text { RELA }\end{array}$ \\
\hline $\begin{array}{l}\text { AGE-RAGE signaling pathway in diabetic } \\
\text { complications }\end{array}$ & 7 & -11.46555508 & AKT1|ICAM1|MAPK1|MAPK8|RELA|TNFIVCAM1 \\
\hline Chagas disease (American trypanosomiasis) & 7 & -11.37271885 & AKT1|IL2|NFKBIAIMAPK1|MAPK8|RELAITNF \\
\hline Toxoplasmosis & 7 & -11.05505232 & AKT1|ALOX5|NFKBIA|MAPK1|MAPK8|RELAITNF \\
\hline Prolactin signaling pathway & 6 & -10.35810608 & AKT1|ESR1|ESR2|MAPK1|MAPK8|RELA \\
\hline Fluid shear stress and atherosclerosis & 7 & -10.35077372 & AKT1|ICAM1|MMP9|MAPK8|RELA|TNF|VCAM1 \\
\hline HTLV-I infection & 8 & -10.24468526 & AKT1|ICAM1|IL2|NFKBIA|MAPK8|RELA|TNF|VCAM1 \\
\hline Influenza A & 7 & -9.746401964 & AKT1IICAM1INFKBIAIMAPK1IMAPK8|RELAITNF \\
\hline NF-kappa B signaling pathway & 6 & -9.543195864 & ICAM1|NFKBIA|PTGS2|RELA|TNF|VCAM1 \\
\hline Endocrine resistance & 6 & -9.51542753 & AKT1|ESR1|ESR2|MMP9|MAPK1|MAPK8 \\
\hline T-cell receptor signaling pathway & 6 & -9.329065252 & AKT1|IL2INFKBIAIMAPK1|RELAITNF \\
\hline Toll-like receptor signaling pathway & 6 & -9.303518428 & AKT1|NFKBIA|MAPK1|MAPK8|RELAITNF \\
\hline Th17 cell differentiation & 6 & -9.228377407 & IL2INFKBIAIMAPK1|MAPK8|RARA|RELA \\
\hline Insulin resistance & 6 & -9.228377407 & AKT1INFKBIAIMAPK8IPTENIRELAITNF \\
\hline Sphingolipid signaling pathway & 6 & -8.970385519 & AKT1|MAPK1|MAPK8|PTEN|RELA|TNF \\
\hline Osteoclast differentiation & 6 & -8.715891959 & AKT1INFKBIA|MAPK1|MAPK8|RELA|TNF \\
\hline Hepatitis C & 6 & -8.695791279 & AKT1INFKBIAIMAPK1|MAPK8|RELA|TNF \\
\hline Apoptosis & 6 & -8.55937549 & AKT1|NFKBIA|MAPK1|MAPK8|RELA|TNF \\
\hline Fc epsilon RI signaling pathway & 5 & -8.326499522 & AKT1|ALOX5|MAPK1|MAPK8ITNF \\
\hline
\end{tabular}




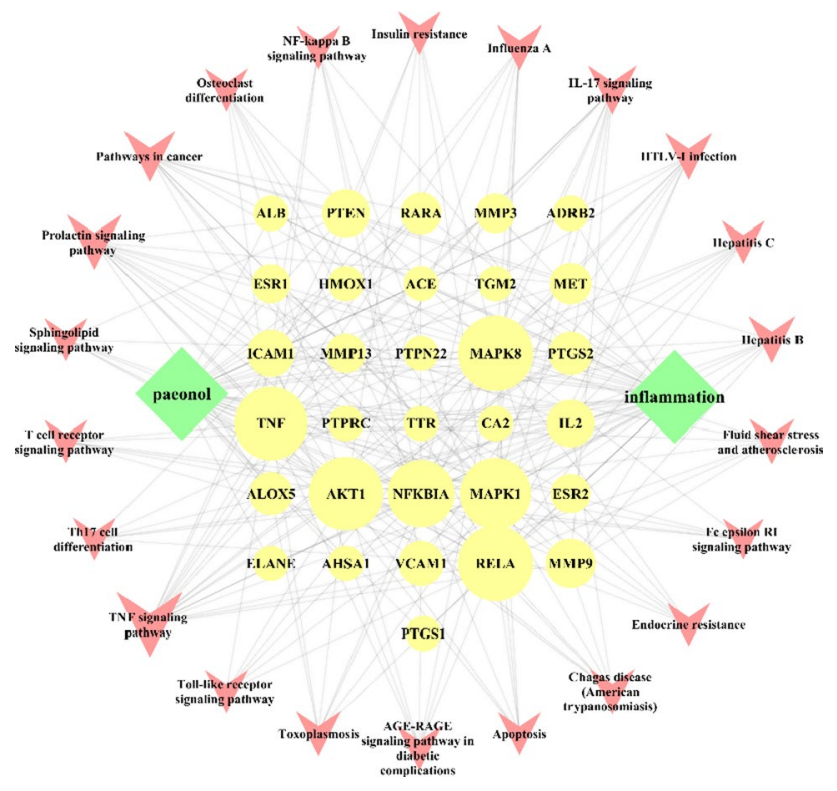

Fig. 4 Target-signaling pathway network of paeonol anti-inflammatory activity. From the inside to the outside: the circle is target, the diamond is paeonol and inflammation, and the inverted triangle is the signaling pathway. Node size: larger nodes indicate more important ones

have good binding activity with these target proteins [19]. In addition, the docking diagram of paeonol with the top three targets is shown in Fig. 5, and bonding types mainly include hydrogen bond and hydrophobic interaction.

\section{Discussion}

Inflammation is a spontaneous defensive response of the human body to "irritant." It usually manifests as redness, heat, swelling, and pain. "Irritant" refers to inflammatory factors, which can be divided into internal and external factors. Internal factors include tissue necrosis, accumulated metabolites, and allergic reactions. External factors include microorganisms (bacteria, viruses, fungi, parasites), physical factors (ultraviolet waves, mechanical damage, temperature), and chemical factors (strong acids, strong alkali, toxic substances). Generally, inflammation is beneficial to the body and helps the body to resist the attack of inflammatory factors. However, excessive inflammatory response can cause serious tissue damage and organ dysfunction [20]. Pruritus and organ damage are common diseases caused by inflammation. In addition, neurodegenerative diseases (Alzheimer's disease, depression, Parkinson's disease), cardiovascular disease, COVID-19, and cancer are also closely related to inflammation [21-23]. At present, nonsteroidal anti-inflammatory drugs are mainly used in the treatment of various inflammatory diseases, but there is a risk of gastrointestinal adverse reactions and hypersensitivity [24-26]. Paeonol has a wide range of pharmacological effects, of which the anti-inflammatory effect is the most important for clinical application, and the concern is that, paeonol has no obvious adverse reactions. Therefore, this study aimed to explore the molecular mechanism of paeonol anti-inflammatory action by network pharmacology and high-throughput molecular docking.

The anti-inflammatory effects of paeonol are mainly manifested in skin inflammation, arthritis, colitis, and organ damage. Paeonol alleviates UV-induced skin inflammation by inhibiting the release of IL-6, MMP-1, and TNF- $\alpha$ [27]. Targeting of the inflammatory factors is one of the important means to treat arthritis. Specifically, IL-1 plays a key role in the occurrence and development of arthritis. The paeonol-related inhibition of IL-1 can reduce the release of PGE2 and NO, thereby ensuring the normal life activities of chondrocytes [28]. The MAPK/ERK/p38 pathway is an important pathway used by paeonol in the treatment of colitis; it is related to the production of inflammatory factors and the clearance of free radicals [29]. Drugs, alcohol, obesity, and emotional agitation are important factors leading to liver injury. Paeonol exerts anti-inflammatory and antioxidant effects through the SIRT1/Nrf2/NF- $\mathrm{KB}$ signaling pathway, thereby reducing alcoholic hepatitis, which suggests that SIRT1 may be a potential drug target for the treatment of inflammation [30]. In China, paeonol has been successfully applied in the treatment of various inflammatory diseases for nearly 50 years [31], and it has achieved good curative effects. At present, paeonol preparations commonly used in clinical practice include paeonol ointment [32], paeonol cream [33], safflower paeonol ointment [34], paeonol injection [35], and compound paeonol dripping pill [36]. However, the poor oral bioavailability of paeonol limits its clinical application.

This study integrated the information from multiple databases and platforms to reveal the anti-inflammatory mechanism of paeonol by network pharmacology and verified the core targets by molecular docking. In the "target-signaling pathway network of paeonol anti-inflammatory action," we screened 22 core targets (e.g., RELA, MAPK8, TNF, AKT1, MAPK1, NFKBIA) and 33 main signaling pathways (e.g., TNF signaling pathway, Prolactin signaling pathway, Pathways in cancer, IL-17 signaling pathway) according to the "degree" value of nodes. The PPI network can find significant genes in the drug-target-disease network, and 23 core proteins such as ELANE, TNF, and MAPK8 were obtained. MAPK is one of the core targets in paeonol targetsignaling pathway network (Fig. 4). It is an important kinase involved in intracellular and extracellular signal transduction. It is reported that pre-oral paeonol in rats can effectively reduce inflammatory diseases, including colitis, and the mechanism is related to the inhibition of the MAPK/ 
Table 2 Characteristic parameters of target-signaling pathway network

\begin{tabular}{|c|c|c|c|}
\hline Type & Name & Degree & Closeness centrality \\
\hline Target & RELA & 22 & 0.613636 \\
\hline Target & MAPK8 & 22 & 0.613636 \\
\hline Target & TNF & 21 & 0.6 \\
\hline Target & AKT1 & 21 & 0.6 \\
\hline Target & MAPK1 & 20 & 0.586957 \\
\hline Target & NFKBIA & 18 & 0.5625 \\
\hline Target & MMP9 & 9 & 0.473684 \\
\hline Target & PTEN & 8 & 0.465517 \\
\hline Target & IL2 & 8 & 0.465517 \\
\hline Target & ICAM1 & 8 & 0.465517 \\
\hline Target & VCAM1 & 7 & 0.457627 \\
\hline Target & PTGS2 & 6 & 0.45 \\
\hline Target & ALOX5 & 6 & 0.45 \\
\hline Target & RARA & 5 & 0.442623 \\
\hline Target & MET & 5 & 0.442623 \\
\hline Target & MMP3 & 4 & 0.435484 \\
\hline Target & MMP13 & 4 & 0.435484 \\
\hline Target & ESR2 & 4 & 0.435484 \\
\hline Target & ESR1 & 4 & 0.435484 \\
\hline Target & TTR & 2 & 0.421875 \\
\hline Target & TGM2 & 2 & 0.421875 \\
\hline Target & PTPRC & 2 & 0.421875 \\
\hline Target & PTPN22 & 2 & 0.421875 \\
\hline Target & PTGS1 & 2 & 0.421875 \\
\hline Target & HMOX1 & 2 & 0.421875 \\
\hline Target & ELANE & 2 & 0.421875 \\
\hline Target & $\mathrm{CA} 2$ & 2 & 0.421875 \\
\hline Target & ALB & 2 & 0.421875 \\
\hline Target & AHSA1 & 2 & 0.421875 \\
\hline Target & ADRB2 & 2 & 0.421875 \\
\hline Target & ACE & 2 & 0.421875 \\
\hline Pathway & TNF signaling pathway & 17 & 0.514286 \\
\hline Pathway & Prolactin signaling pathway & 12 & 0.469565 \\
\hline Pathway & Pathways in cancer & 10 & 0.453782 \\
\hline Pathway & IL-17 signaling pathway & 9 & 0.446281 \\
\hline Pathway & Hepatitis B & 8 & 0.439024 \\
\hline Pathway & HTLV-I infection & 8 & 0.439024 \\
\hline Pathway & $\begin{array}{l}\text { AGE-RAGE signaling pathway in diabetic com- } \\
\text { plications }\end{array}$ & 7 & 0.432 \\
\hline Pathway & Chagas disease (American trypanosomiasis) & 7 & 0.432 \\
\hline Pathway & Toxoplasmosis & 7 & 0.432 \\
\hline Pathway & Fluid shear stress and atherosclerosis & 7 & 0.432 \\
\hline Pathway & Influenza A & 7 & 0.432 \\
\hline Pathway & NF-kappa B signaling pathway & 6 & 0.418605 \\
\hline Pathway & Endocrine resistance & 6 & 0.418605 \\
\hline Pathway & $\mathrm{T}$ cell receptor signaling pathway & 6 & 0.425197 \\
\hline Pathway & Toll-like receptor signaling pathway & 6 & 0.425197 \\
\hline Pathway & Th17 cell differentiation & 6 & 0.425197 \\
\hline Pathway & Insulin resistance & 6 & 0.425197 \\
\hline Pathway & Sphingolipid signaling pathway & 6 & 0.425197 \\
\hline Pathway & Osteoclast differentiation & 6 & 0.425197 \\
\hline
\end{tabular}


Table 2 (continued)

\begin{tabular}{llll}
\hline Type & Name & Degree & Closeness centrality \\
\hline Pathway & Hepatitis C & 6 & 0.425197 \\
Pathway & Apoptosis & 6 & 0.425197 \\
Pathway & Fc epsilon RI signaling pathway & 5 & 0.418605 \\
\hline
\end{tabular}

Table 3 Optimal binding energies between paeonol and core targets

\begin{tabular}{|c|c|c|c|c|}
\hline Rank & Target name & Protein name & PDB ID & Score $(\mathrm{kcal} / \mathrm{mol})$ \\
\hline 1 & MMP9 & Matrix metalloproteinase- 9 & $6 \mathrm{ESM}$ & -7.4 \\
\hline 2 & MMP13 & Collagenase 3 & $5 \mathrm{~B} 5 \mathrm{O}$ & -7.1 \\
\hline 3 & ALB & Albumin & 6YG9 & -6.8 \\
\hline 4 & PTGS2 & Prostaglandin G/H synthase 2 & $5 \mathrm{~F} 19$ & -6.7 \\
\hline 5 & PTGS1 & Prostaglandin G/H synthase 1 & $6 \mathrm{Y} 3 \mathrm{C}$ & -6.7 \\
\hline 6 & MMP3 & Stromelysin-1 & $1 \mathrm{HY} 7$ & -6.6 \\
\hline 7 & RARA & Retinoic acid receptor alpha & $3 \mathrm{KMR}$ & -6.4 \\
\hline 8 & MET & Hepatocyte growth factor receptor & $4 \mathrm{R} 1 \mathrm{~V}$ & -6.3 \\
\hline 9 & ALOX5 & Polyunsaturated fatty acid 5-lipoxygenase & 3V98 & -6.2 \\
\hline 10 & MAPK8 & Mitogen-activated protein kinase 8 & 2XRW & -6 \\
\hline 11 & ESR1 & Estrogen receptor & $6 \mathrm{VIG}$ & -5.8 \\
\hline 12 & PTEN & $\begin{array}{l}\text { Phosphatidylinositol 3,4,5-trisphosphate 3-phos- } \\
\text { phatase and dual-specificity protein phosphatase } \\
\text { PTEN }\end{array}$ & $1 \mathrm{D} 5 \mathrm{R}$ & -5.7 \\
\hline 13 & MAPK1 & Mitogen-activated protein kinase 1 & $4 Z Z N$ & -5.5 \\
\hline 14 & ESR2 & Estrogen receptor beta & 3OLL & -5.4 \\
\hline 15 & TTR & Transthyretin & 4D7B & -5.3 \\
\hline 16 & IL2 & Interleukin-2 & 4NEJ & -5.1 \\
\hline 17 & ELANE & Neutrophil elastase & $5 \mathrm{ABW}$ & -5.1 \\
\hline 18 & TNF & Tumor necrosis factor & $5 \mathrm{UUI}$ & -4.9 \\
\hline 19 & AKT1 & RAC-alpha serine/threonine-protein kinase & $1 \mathrm{UNQ}$ & -4.7 \\
\hline 20 & NFKBIA & NF-kappa-B inhibitor alpha & $6 \mathrm{Y} 1 \mathrm{~J}$ & -4.7 \\
\hline 21 & ICAM1 & Intercellular adhesion molecule 1 & 1IAM & -4.7 \\
\hline 22 & VCAM1 & Vascular cell adhesion protein 1 & $1 \mathrm{VCA}$ & -4.6 \\
\hline 23 & RELA & Transcription factor $\mathrm{p} 65$ & $6 \mathrm{NV} 2$ & -3.4 \\
\hline
\end{tabular}

ERK/p38 signaling pathway [29]. TNF is one of the most important signaling pathways in paeonol target-signaling pathway network (Fig. 4). As a cytokine closely related to inflammation, it is a key indicator of many diseases. Paeonol can affect the expression of TNF- $\alpha$, and TNF- $\alpha$ can activate $\mathrm{NF}-\kappa \mathrm{B}$, thereby resulting in anti-inflammatory response. In addition, the functional modules including MAPK1, RARA, and ESR2 have important biological significance for the treatment of inflammation [37, 38]. According to the GO enrichment analysis results, the biological processes of paeonol anti-inflammatory action mainly involve response to inflammatory factors, regulation of DNA-binding transcription factor activity, and response to oxidative stress. Therefore, paeonol may play an anti-inflammatory role by regulating the related targets of these biological processes. The KEGG pathway analysis showed that paeonol anti-inflammation-related pathways mainly involved TNF signaling pathway and IL-17 signaling pathway. Allergic dermatitis is an inflammatory reaction of the skin caused by excessive immunity, and it belongs to allergic reactions. It has been reported that paeonol can reduce the release of $\operatorname{lgE}$ by regulating TNF and histamine, thereby playing an antiallergic role [39]. In addition, paeonol at different doses (200 and $400 \mathrm{mg} / \mathrm{kg}$ ) has shown certain therapeutic effects on colitis in rats; specifically, it can block IL-17 signaling pathway and promote TGF- $\beta 1$ production, thereby improving the pathological score of colon tissue [40]. Thus, TNF and IL-17-related signaling pathways may be an important molecular mechanism by which paeonol exerts its antiinflammatory effects.

Molecular docking takes the active center of the target as the "docking pocket" and is an important means to verify the binding ability between drugs and targets, which can save a lot of time, manpower, material, and financial resources. 
A
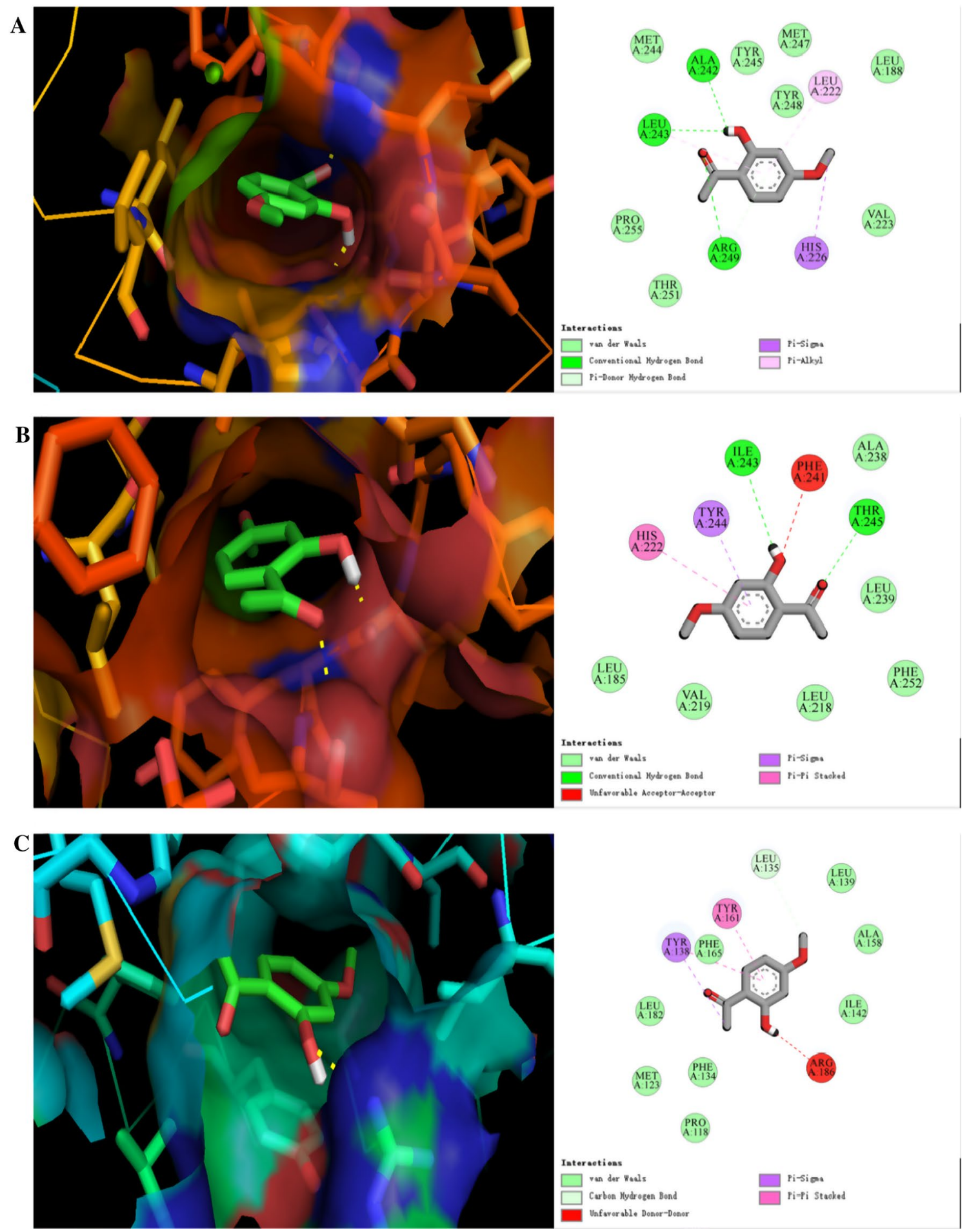

Fig. 5 Docking diagram of paeonol with the top three targets. a paeonol with MMP9, $\mathbf{b}$ paeonol with MMP13, and $\mathbf{c}$ paeonol with ALB; 3D diagram on the left and the 2D diagram on the right show the positions of active pockets and the types of interactions

Molecular docking results showed that the docking energy values were lower than 0 , of which $74 \%$ were lower than -5 , indicating that paeonol has good affinity to these core targets. Importantly, MMP9 has the strongest binding affinity to paeonol. Among all the targets in the PPI network, MMP9, which can maintain the dynamic balance of extracellular matrix, is the target with the highest binding affinity to paeonol. In vitro experiments have shown that paeonol could 
inhibit the growth, reproduction, and migration of tumor cells by regulating MMP9 in a concentration-dependent manner, and the mechanism was related to inflammationrelated pathways such as NF- $\mathrm{KB}$ signaling pathway [41]. Therefore, it is speculated that MMP9 may be one of the important targets for paeonol to exert its anti-inflammatory effect.

In conclusion, paeonol exerts anti-inflammatory effects by acting on 22 targets and 33 signaling pathways, and it is closely related to the response to inflammatory factors, regulation of DNA-binding transcription factor activity, and response to oxidative stress. These potential targets have certain reference value for the study of paeonol targeted drugs.

Acknowledgements This work is supported by the National Natural Science Foundation of China (No.82003715), Projects of Medical and Health Technology Development Program in Shandong Province (2019WS557), Key R \& D project of Shandong Province (2020CXGC010505).

Authors' contributions J-HQ, F-XD, and X-LW have participated in the experiment and manuscript. J-HQ and F-XD have contributed equally to this work. X-LW have checked the contents of the manuscript and revised the language. In addition, X-LW gave guidance and effective suggestions on the overall manuscript design, data processing and discussion revision.

Availability of data and material The data that support the findings of this study are available from the corresponding author upon reasonable request.

\section{Declarations}

Conflict of interest The authors declare that they have no conflicts of interest.

\section{References}

1. Xue P, Wang Y, Zeng F, Xiu R, Chen J, Guo J, Yuan P, Liu L, Xiao J, Lu H, Wu D, Pan H, Lu M, Zhu F, Shi F, Duan Q (2017) Paeonol suppresses solar ultraviolet-induced skin inflammation by targeting T-LAK cell-originated protein kinase. Oncotarget 8(16):27093-27104. https://doi.org/10.18632/oncotarget.15636

2. Meng Y, Liu Z, Zhai C, Di T, Zhang L, Zhang L, Xie X, Lin Y, Wang N, Zhao J, Wang Y, Li P (2019) Paeonol inhibits the development of 1-chloro-2,4-dinitrobenzene-induced atopic dermatitis via mast and T cells in BALB/c mice. Mol Med Rep 19(4):32173229. https://doi.org/10.3892/mmr.2019.9985

3. Fu J, Yu L, Luo J, Huo R, Zhu B (2018) Paeonol induces the apoptosis of the SGC-7901 gastric cancer cell line by downregulating ERBB2 and inhibiting the NF- $\mathrm{KB}$ signaling pathway. Int J Mol Med 42(3):1473-1483. https://doi.org/10.3892/ijmm.2018.3704

4. Yuan X, Chen J, Dai M (2016) Paeonol promotes microRNA-126 expression to inhibit monocyte adhesion to ox-LDL-injured vascular endothelial cells and block the activation of the PI3K/Akt/ NF-אB pathway. Int J Mol Med 38(6):1871-1878. https://doi.org/ 10.3892/ijmm.2016.2778

5. Jin H, Wang M, Wang J, Cao H, Niu W, Du L (2020) Paeonol attenuates isoflurane anesthesia-induced hippocampal neurotoxicity via modulation of JNK/ERK/P38MAPK pathway and regulates histone acetylation in neonatal rat. J Matern Fetal Neonatal Med 33(1):81-91. https://doi.org/10.1080/14767058. 2018.1487396

6. Zhu XL, Chen JJ, Han F, Pan C, Zhuang TT, Cai YF, Lu YP (2018) Novel antidepressant effects of Paeonol alleviate neuronal injury with concomitant alterations in BDNF, Rac1 and RhoA levels in chronic unpredictable mild stress rats. Psychopharmacology 235(7):2177-2191. https://doi.org/10.1007/s00213-018-4915-7

7. Harada M, Yamashita A (1969) Pharmacological studies on the root bark of paeonia moutan. I. Central effects of paeonol. Yakugaku zasshi J Pharm Soc Japan 89(9):1205-1211. https://doi.org/ 10.1248/yakushi1947.89.9_1205

8. Ru J, Li P, Wang J, Zhou W, Li B, Huang C, Li P, Guo Z, Tao W, Yang Y, Xu X, Li Y, Wang Y, Yang L (2014) TCMSP: a database of systems pharmacology for drug discovery from herbal medicines. J Cheminform 6:13. https://doi.org/10.1186/ 1758-2946-6-13

9. Daina A, Michielin O, Zoete V (2019) SwissTargetPrediction: updated data and new features for efficient prediction of protein targets of small molecules. Nucleic Acids Res 47(W1):W357w364. https://doi.org/10.1093/nar/gkz382

10. Consortium U (2019) UniProt: a worldwide hub of protein knowledge. Nucleic Acids Res 47(D1):D506-D515. https://doi.org/10. 1093/nar/gky1049

11. Amberger JS, Bocchini CA, Schiettecatte F, Scott AF, Hamosh A (2015) OMIM.org: Online Mendelian Inheritance in Man (OMIM®), an online catalog of human genes and genetic disorders. Nucleic Acids Res 43(Database issue): D789-D798. https:// doi.org/10.1093/nar/gku1205

12. Wishart DS, Feunang YD, Guo AC, Lo EJ, Marcu A, Grant JR, Sajed T, Johnson D, Li C, Sayeeda Z, Assempour N, Iynkkaran I, Liu Y, Maciejewski A, Gale N, Wilson A, Chin L, Cummings R, Le D, Pon A, Knox C, Wilson M (2018) DrugBank 5.0: a major update to the DrugBank database for 2018. Nucleic Acids Res 46(D1): D1074-D1082. https://doi.org/10.1093/nar/gkx1037

13. Wang Y, Zhang S, Li F, Zhou Y, Zhang Y, Wang Z, Zhang R, Zhu J, Ren Y, Tan Y, Qin C, Li Y, Li X, Chen Y, Zhu F (2020) Therapeutic target database 2020: enriched resource for facilitating research and early development of targeted therapeutics. Nucleic Acids Res 48(D1):D1031-d1041. https://doi.org/10.1093/ nar/gkz981

14. Bardou P, Mariette J, Escudié F, Djemiel C, Klopp C (2014) jvenn: an interactive Venn diagram viewer. BMC Bioinform 15(1):293. https://doi.org/10.1186/1471-2105-15-293

15. Szklarczyk D, Gable AL, Lyon D, Junge A, Wyder S, HuertaCepas J, Simonovic M, Doncheva NT, Morris JH, Bork P, Jensen LJ, Mering CV (2019) STRING v11: protein-protein association networks with increased coverage, supporting functional discovery in genome-wide experimental datasets. Nucleic Acids Res 47(D1):D607-d613. https://doi.org/10.1093/nar/gky1131

16. Trott O, Olson AJ (2010) AutoDock Vina: improving the speed and accuracy of docking with a new scoring function, efficient optimization, and multithreading. J Comput Chem 31(2):455-461. https://doi.org/10.1002/jcc.21334

17. Berman HM, Westbrook J, Feng Z, Gilliland G, Bhat TN, Weissig H, Shindyalov IN, Bourne PE (2000) The protein data bank. Nucleic Acids Res 28(1):235-242. https://doi.org/10.1093/nar/ 28.1.235

18. Schrodinger LLC (2015) The PyMOL molecular graphics system, version 1.8

19. Bai YL, Zhang JF, Sha ZJ, Zhu N, Huang XL, Li ZY (2021) Analysis of the potential molecular mechanism of Bawei Sanxiang Powder in the treatment of chronic heart failure based on network pharmacology and molecular docking. Chin J Chin Mater Med $1-14$ 
20. Huang C, Wang Y, Li X, Ren L, Zhao J, Hu Y, Zhang L, Fan G, Xu J, Gu X, Cheng Z, Yu T, Xia J, Wei Y, Wu W, Xie X, Yin W, Li H, Liu M, Xiao Y, Gao H, Guo L, Xie J, Wang G, Jiang R, Gao Z, Jin Q, Wang J, Cao B (2020) Clinical features of patients infected with 2019 novel coronavirus in Wuhan. China Lancet 395(10223):497-506. https://doi.org/10.1016/s0140-6736(20) 30183-5

21. Dorothée G (2018) Neuroinflammation in neurodegeneration: role in pathophysiology, therapeutic opportunities and clinical perspectives. J Neural Transm (Vienna) 125(5):749-750. https://doi. org/10.1007/s00702-018-1880-6

22. Hadjadj J, Yatim N, Barnabei L, Corneau A, Boussier J, Smith N, Péré H, Charbit B, Bondet V, Chenevier-Gobeaux C, Breillat P, Carlier N, Gauzit R, Morbieu C, Pène F, Marin N, Roche N, Szwebel TA, Merkling SH, Treluyer JM, Veyer D, Mouthon L, Blanc C, Tharaux PL, Rozenberg F, Fischer A, Duffy D, RieuxLaucat F, Kernéis S, Terrier B (2020) Impaired type I interferon activity and inflammatory responses in severe COVID-19 patients. Science (New York, NY) 369(6504):718-724. https://doi.org/10. 1126/science.abc6027

23. Weber B, Liao KP, DiCarli M, Blankstein R (2021) Cardiovascular disease prevention in individuals with underlying chronic inflammatory disease. Curr Opin Cardiol 36(5):549-555. https:// doi.org/10.1097/hco.0000000000000877

24. Dona I, Salas M, Perkins JR, Barrionuevo E, Gaeta F, CornejoGarcia JA, Campo P, Torres MJ (2016) Hypersensitivity reactions to non-steroidal anti-inflammatory drugs. Curr Pharm Des 22(45):6784-6802. https://doi.org/10.2174/138161282266616 0928142814

25. Perkins JR, Sanak M, Canto G, Blanca M, Cornejo-García JA (2015) Unravelling adverse reactions to NSAIDs using systems biology. Trends Pharmacol Sci 36(3):172-180. https://doi.org/10. 1016/j.tips.2014.12.003

26. Polimeni G, Salvo F, Cutroneo P, Morreale I, Patrizio Caputi A (2006) Adverse reactions induced by NSAIDs and antibacterials: analysis of spontaneous reports from the Sicilian regional database. Drug Saf 29(5):449-459. https://doi.org/10.2165/00002 018-200629050-00006

27. Sun Z, Du J, Hwang E, Yi TH (2018) Paeonol extracted from Paeonia suffruticosa Andr. ameliorated UVB-induced skin photoaging via DLD/Nrf2/ARE and MAPK/AP-1 pathway. Phytother Res 32(9): 1741-1749. https://doi.org/10.1002/ptr.6100

28. Liu M, Zhong S, Kong R, Shao H, Wang C, Piao H, Lv W, Chu X, Zhao Y (2017) Paeonol alleviates interleukin-1 $\beta$-induced inflammatory responses in chondrocytes during osteoarthritis. Biomed Pharmacother 95: 914-921. https://doi.org/10.1016/j.biopha.2017. 09.011

29. Jin X, Wang J, Xia ZM, Shang CH, Chao QL, Liu YR, Fan HY, Chen DQ, Qiu F, Zhao F (2016) Anti-inflammatory and Antioxidative Activities of paeonol and its metabolites through blocking MAPK/ERK/p38 signaling pathway. Inflammation 39(1):434446. https://doi.org/10.1007/s10753-015-0265-3

\section{Authors and Affiliations}

\section{Jian-hong Qi ${ }^{1}\left(\mathbb{D} \cdot\right.$ Fang-xu Dong ${ }^{2} \cdot$ Xiao-long Wang ${ }^{3,4,5}$}

Jian-hong Qi

qjh951024@163.com

$\triangle$ Xiao-long Wang

wangxl_hl@126.com
30. Sun X, Wang P, Yao LP, Wang W, Gao YM, Zhang J, Fu YJ (2018) Paeonol alleviated acute alcohol-induced liver injury via SIRT1/Nrf2/NF- $\kappa B$ signaling pathway. Environ Toxicol Pharmacol 60:110-117. https://doi.org/10.1016/j.etap.2018.04.016

31. Shanghai First Pharmaceutical Factory (1973) Development and clinical observation of Xu Changqing injection. Chin Tradit Herbal Drugs 07:7-8

32. Lu LG, Wu SQ, Liang D (2013) Study on the tolerance of safflower paeonol ointment in phase I clinical trial. Chin J Chin Mater Med 38(01):123-126

33. Yu CR (2014) Observation on the efficacy of paeonol ointment in the treatment of 64 cases of eczema and dermatitis. China Health Industry 11(01):58-59

34. Xiao ZG, Li SL, Wu B, Zhong CQ (2018) 80 cases of gluteal epithelial neuritis treated by massage combined with paeonol injection. Chin J Tradit Med Traumatol Orthop 26(03):61-62, 65

35. Wang HM, Cai XW, Xu M, He X, Hao ZQ (2019) Effects of paeonol on periodontal status in elderly patients. Chin J Geriatric Dent 17(03): 158-162

36. Wang X, Hu DY, Sha O (2008) Effect of compound paeonol dripping pill on levels of plasma inflammatory mediators in patients with unstable angina. Chin J Integ Trad West Med 28(5):395-398

37. Chen G, Jia P, Yin ZY, Kong SZ, Xiang ZB, Zheng XX (2019) Paeonol ameliorates monosodium urate-induced arthritis in rats through inhibiting nuclear factor- $\mathrm{kB}$-mediated proinflammatory cytokine production. Phytotherapy Res PTR 33(11):2971-2978. https://doi.org/10.1002/ptr.6472

38. Liu N, Feng X, Wang W, Zhao X, Li X (2017) Paeonol protects against TNF- $\alpha$-induced proliferation and cytokine release of rheumatoid arthritis fibroblast-like synoviocytes by upregulating FOXO3 through inhibition of miR-155 expression. Inflamm Res 66(7):603-610. https://doi.org/10.1007/s00011-017-1041-7

39. Kim SH, Kim SA, Park MK, Kim SH, Park YD, Na HJ, Kim HM, Shin MK, Ahn KS (2004) Paeonol inhibits anaphylactic reaction by regulating histamine and TNF-alpha. Int Immunopharmacol 4(2):279-287. https://doi.org/10.1016/j.intimp.2003.12.013

40. Zong SY, Pu YQ, Xu BL, Zhang T, Wang B (2017) Study on the physicochemical properties and anti-inflammatory effects of paeonol in rats with TNBS-induced ulcerative colitis. Int Immunopharmacol 42:32-38. https://doi.org/10.1016/j.intimp.2016.11. 010

41. Lyu ZK, Li CL, Jin Y, Liu YZ, Zhang X, Zhang F, Ning LN, Liang ES, Ma M, Gao W, Zhang MX, Liu DS (2017) Paeonol exerts potential activities to inhibit the growth, migration and invasion of human gastric cancer BGC823 cells via downregulating MMP-2 and MMP-9. Mol Med Rep 16(5):7513-7519. https://doi.org/10. 3892/mmr.2017.7576

Publisher's Note Springer Nature remains neutral with regard to jurisdictional claims in published maps and institutional affiliations.

1 College of Pharmacy, Shandong University of Traditional Chinese Medicine, Changqing University of Science and Technology Park, Changqing District, Jinan 250355, Shandong, China

2 College of Foreign Languages, Shandong University of Traditional Chinese Medicine, Jinan, Shandong, China 
3 The Experiment Center, Shandong University of Traditional Chinese Medicine, Changqing University Science \& Technology Park, Changqing District, Jinan 250355, Shandong, China

4 Key Laboratory of Traditional Chinese Medicine Classical Theory, Ministry of Education, Shandong University of Traditional Chinese Medicine, Jinan 250355, China
5 Shandong Provincial Key Laboratory of Traditional Chinese Medicine for Basic Research, Shandong University of Traditional Chinese Medicine, Jinan 250355, China 